\title{
TRaCE+: Ensemble inference of gene regulatory networks from transcriptional expression profiles of gene knock-out experiments
}

\author{
S.M. Minhaz Ud-Dean ${ }^{1,2}$, Sandra Heise ${ }^{3}$, Steffen Klamt $^{3}$ and Rudiyanto Gunawan ${ }^{1,2^{*}}$
}

\begin{abstract}
Background: The inference of gene regulatory networks (GRNs) from transcriptional expression profiles is challenging, predominantly due to its underdetermined nature. One important consequence of underdetermination is the existence of many possible solutions to this inference. Our previously proposed ensemble inference algorithm TRaCE addressed this issue by inferring an ensemble of network directed graphs (digraphs) using differential gene expressions from gene knock-out (KO) experiments. However, TRaCE could not deal with the mode of the transcriptional regulations (activation or repression), an important feature of GRNs.

Results: In this work, we developed a new algorithm called TRaCE+ for the inference of an ensemble of signed GRN digraphs from transcriptional expression data of gene KO experiments. The sign of the edges indicates whether the regulation is an activation (positive) or a repression (negative). TRaCE+ generates the upper and lower bounds of the ensemble, which define uncertain regulatory interactions that could not be verified by the data. As demonstrated in the case studies using Escherichia coli GRN and 100-gene gold-standard GRNs from DREAM 4 network inference challenge, by accounting for regulatory signs, TRaCE+ could extract more information from the $\mathrm{KO}$ data than TRaCE, leading to fewer uncertain edges. Importantly, iterating TRaCE+ with an optimal design of gene KOs could resolve the underdetermined issue of GRN inference in much fewer KO experiments than using TRaCE.

Conclusions: TRaCE+ expands the applications of ensemble GRN inference strategy by accounting for the mode of the gene regulatory interactions. In comparison to TRaCE, TRaCE+ enables a better utilization of gene KO data, thereby reducing the cost of tackling underdetermined GRN inference. TRaCE+ subroutines for MATLAB are freely available at the following website: http://www.cabsel.ethz.ch/tools/trace.html.
\end{abstract}

Keywords: Gene regulatory network, Network inference, Design of experiments, Signed directed graph, Transitive reduction

\section{Background}

The central dogma of molecular biology describes the process by which genetic information flows linearly from deoxyribonucleic acid (DNA) to ribonucleic acid (RNA) to proteins through the process of transcription and translation [1]. This dogma has guided research on the causes of

\footnotetext{
*Correspondence: rudi.gunawan@chem.ethz.ch

'Institute for Chemical and Bioengineering, ETH Zurich, Zurich, Switzerland

${ }^{2}$ Swiss Institute of Bioinformatics, Lausanne, Switzerland

Full list of author information is available at the end of the article
}

cellular phenotype and diseases since its inception in 1956. However, such reductionist view has been continually challenged in the post-genomic era, during which we also saw the rise of systems biology and the use of networks to understand biology at all levels. One prominent example of such networks is a gene regulatory network (GRN), which describes how the protein product(s) of one gene activates or inhibits the transcription of other genes. The knowledge of this network and its pathological alterations could lead to, among other things, a better 
understanding of how cell phenotype arise from the interactions among cell constituents, and to novel treatments and drugs for genetic diseases such as cancer [2].

The inference of GRN has received great attention from the systems biology community, especially with the ubiquity of gene transcriptional profiling using DNA microarray chip and RNA sequencing. A multitude of network inference methods now exist in the literature for the identification of GRN structure from gene transcriptional expression data [3-9]. These methods adapted concepts and techniques from multiple disciplines such as information theory, statistics, machine learning and systems theory. The large number of methods called for the establishment of gold standard data for a fair and objective assessment. The DREAM (Dialogue for Reverse Engineering, Assessment and Methods) project materialized as an answer [10], and the GRN inference became a topic in several community-wide challenges within this project.

The results of several GRN inference challenges signified a fundamental issue in this inference. For example, many of the algorithms employed by participants in DREAM 4 in silico network inference challenge did not perform well in inferring small to moderately-sized GRNs (10-100 genes). This poor performance was observed despite the availability of transcriptional expression data of all genes from the complete set of single-gene knock-out (KO) experiments, i.e. deleting each and every gene, one at a time $[8,9,11]$. The network inference challenge further showed that distinguishing direct and indirect regulations was one of the common weaknesses among the participating methods. As we demonstrated recently [12], these difficulties arose because the above GRN inference problems were underdetermined, i.e. the GRNs were not inferable and there could in fact exist an ensemble of GRN structures that agreed with the gene KO data.

We previously developed TRaCE (Transitive Reduction and Closure Ensemble) for constructing an ensemble of GRN structures that are consistent with the input transcriptional expression data of all genes in the network from gene $\mathrm{KO}$ experiments [12]. This ensemble represented the uncertainty in the GRN inference. In developing $\mathrm{TRaCE}$, we employed directed graphs to model the GRNs, where nodes in such graphs describe the genes and directed edges describes the gene regulations. Following the GRN inference formulation in DREAM challenges, we previously ignored the signs of the edges and sought only to establish the existence of gene regulations. Nevertheless, the edge signs in a GRN digraph are often of great interest and significance as they indicate the modes of the gene regulations. Here, a positive edge reflects an activation, while a negative edge describes a repression. Several notable network inference algorithms such as TRANSitive reduction for WEighted Signed Digraphs (TRANSWESD) [13] and
Local Transitive Reduction (LTR) [14] previously considered the inference of GRN digraph with signed (and weighted) edges. However, these algorithms were not designed for inferring an ensemble of GRN structures.

In this work, we addressed the aforementioned drawback of TRaCE by developing a new ensemble inference method, called TRaCE+. The new method uses a signed digraph model of the GRN, i.e. the edges have signs. Like $\mathrm{TRaCE}, \mathrm{TRaCE}+$ generates the upper and lower bounds of the ensemble, but in the form of signed digraphs. The ensemble bounds from TRaCE+ are also compatible with our recent optimal design of gene $\mathrm{KO}$ strategy called REDUCE [15]. We demonstrated the advantages of TRaCE+ over TRaCE in the ensemble inference of Escherichia coli GRN and in the iterative inference of 100-gene gold standard GRNs from DREAM 4 in silico network inference challenge.

\section{Methods \\ Definitions}

In this section we provide basic concepts of graph theory that are relevant to the development of TRaCE+. A graph $G$ is defined by the pair $(V(G), E(G))$ where $V(G)$ denotes the set of vertices or nodes and $E(G) \subseteq$ $V(G) \times V(G)$ denotes the set of edges. The number of nodes $n(G)$ and edges $m(G)$ are called the order and size of the graph, respectively. In a directed graph, an edge is defined by an ordered pair of nodes $(i, j)$ denoting the edge direction, pointing from node $i$ to node $j$. Here, node $i$ is called a parent of node $j$, while node $j$ is called a child of node $i$. The edge $(i, j)$ is also said to be incident to nodes $i$ and $j$. Finally, a signed digraph $G^{+}=\left(V\left(G^{+}\right), E\left(G^{+}\right), S\left(G^{+}\right)\right)$is the digraph $\left(V\left(G^{+}\right), E\left(G^{+}\right)\right)$ with an edge mapping $S: E \rightarrow\{+,-\}$ that assigns a positive or negative sign to each edge.

A directed path in a digraph $G$ is a sequence of nodes $v_{1}, v_{2}, v_{3}, \ldots v_{n}$ such that $\left(v_{1}, v_{2}\right),\left(v_{2}, v_{3}\right), \ldots\left(v_{n-1}, v_{n}\right) \in$ $E(G)$. The number of edges in a directed path is called the path length. The first node of a directed path is called the start node, and the last node is called the end node. A directed cycle is a directed path where the start node and the end node are the same. A directed acyclic graph (DAG) is a digraph which does not contain any directed cycle. A node $v$ is said to be accessible from another node $u$ if there exist a directed path from node $u$ to node $v$. In this case, node $u$ is an ancestor of node $v$, and node $v$ is a descendant of node $u$. The $a d$ jacency matrix of a digraph $G$ is a $n(G) \times n(G)$ matrix where the $(u, v)$-th element is 1 if there exists a directed edge from node $u$ to node $v$ in $G$, and 0 otherwise. Meanwhile, the accessibility matrix of a digraph $G$ is a $n(G) \times n(G)$ matrix where the $(u, v)$-th element is 1 if there exists a directed path from node $u$ to node $v$ in $G$, and 0 otherwise. Multiple digraphs can have the same 
accessibility matrix, among which the digraph with the fewest edges is called the transitive reduction.

In the following sections, we focus on the inference of GRN structure in the form of a signed digraph. We denote the GRN of interest as $G_{\varnothing}^{+}$, which is also referred to as the wild-type GRN. In such a graph, the nodes represent the genes, and the signed directed edges indicate the gene regulations. A positive (negative) edge pointing from gene $i$ to gene $j$ implies that the products of gene $i$ upregulates (represses) the expression of gene $j$. Figure 1a gives an example of a signed digraph of a GRN with 5 genes $(n(G)=5)$ and 7 gene regulatory edges $(m(G)=7)$. Three of the gene regulations are negative (repressions), while four are positive (upregulations). Here, gene $C$ is a parent of genes $D$ and $E$, while gene $D$ is a child of genes $C, B$ and $E$. Meanwhile, gene $A$ is an ancestor of the genes $C, D$ and $E$, while gene $E$ is a descendant of the genes $A, B, C$ and $D$. We further denote the GRN corresponding to knocking-out (deleting) a set of genes $V_{K O} \subset V\left(G_{\varnothing}^{+}\right)$by $G_{V_{K O}}^{+}$. Figure $1 \mathrm{~b}$ illustrates the network $G_{\mathrm{D}}^{+}$, where all edges incident to gene $D$ have been deleted from the GRN $G_{\varnothing}^{+}$in Fig. 1a.

\section{TRaCE+}

An important consequence of the underdetermined nature of GRN inference is the existence of multiple solutions to the inference problem. In order to deal with this consequence, we previously developed an ensemble inference algorithm called TRaCE [12]. In the development of TRaCE and TRaCE+, we recognized a fundamental limitation in using steady state transcriptional expression data of gene KO experiments for GRN inference. This limitation relates to the inability to differentiate direct and indirect gene regulations from such expression data. For example, we expect that knocking out a transcription factor (TF) gene $u$ would lead to steady-state differential expressions among genes that directly regulated by that TF, as well as genes that are indirectly regulated by $u$ through the involvement of other TFs. Following the definitions above, genes which show differential expression upon knocking out gene $u$ thus correspond to all nodes that are accessible from node $u$. Given steady state transcriptional expression data from the complete set of single-gene KOs, one could obtain at most the accessibility relationship (matrix) among the genes.

In $\mathrm{TRaCE}$, we seek to identify the ensemble (family) of GRN digraphs that are consistent with steady state differential gene expressions in $\mathrm{KO}$ experiments. The true GRN is thus expected to belong to this ensemble. More specifically, TRaCE generates two digraphs: the ensemble upper bound $G^{U}$ and the ensemble lower bound $G^{L}$, which compactly represent the entire ensemble. The upper bound $G^{U}$ represents the largest digraph in the ensemble, i.e. one with the most gene regulations or edges. Edges in $G^{U}$ may include both direct and indirect gene regulations. Meanwhile, the lower bound $G^{L}$ is constructed from $G^{U}$ by removing all edges which can be explained by an indirect gene regulation. Therefore, any GRN in the ensemble must contain all edges in the lower bound $G^{L}$ and may include some or all the edges in the upper bound $G^{U}$. The set of gene regulatory edges that

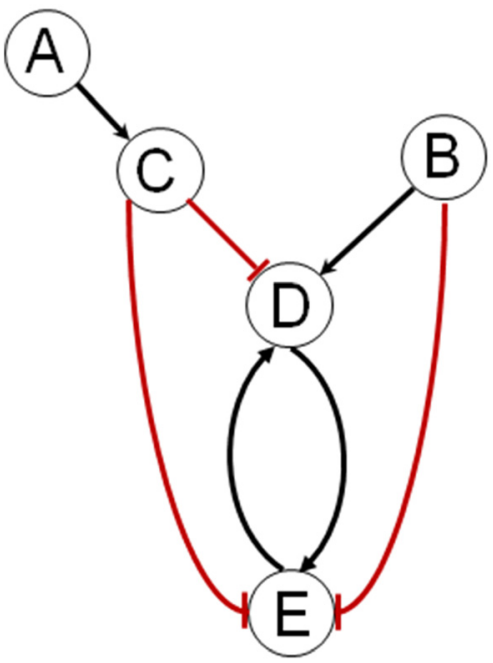

(a)

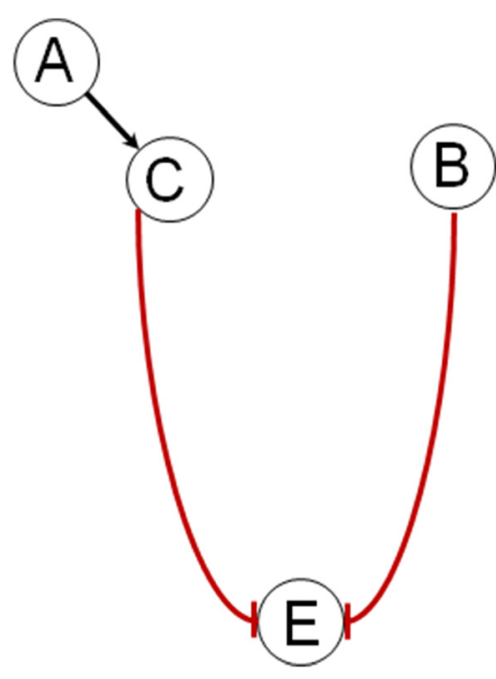

(b)

Fig. 1 Illustration of signed digraph GRNs. a Example of a signed digraph $G_{\varnothing}^{+}$. The pointed black arrows indicate positive regulations while the flathead red arrows represent negative regulations. b The network $G_{\{D\}}^{+}$in which gene D has been deleted 
belong to the upper bound $G^{U}$ but are missing from the lower bound $G^{L}$, denoted by $E_{L}$, are appropriately called uncertain edges. These edges are uncertain since their existence could not be verified by the available data. Furthermore, the number of uncertain edges reflects the degree of uncertainty in the GRN inference. Importantly, by knowing which edges are uncertain, we could optimize $\mathrm{KO}$ experiments in order to maximize the number of edge verifications [15].

One of the biggest drawbacks of TRaCE is its disregard of the signs (modes) of the regulatory edges. For this reason, we have developed TRaCE+. Like TRaCE, $\mathrm{TRaCE}+$ generates the upper and lower bounds of the ensemble, but with signed edges (i.e., these bounds are signed digraphs). TRaCE+ comprises two main algorithms for (1) constructing the ensemble upper and lower bounds using data from the complete set of single-gene KOs, and (2) updating the ensemble bounds using additional gene $\mathrm{KO}$ data. Below, we provide more details of the two algorithms.

\section{Construction of upper bound from single-gene KOs}

In constructing the upper and lower bounds of the ensemble for $\mathrm{TRaCE}+$, we start by putting together the accessibility matrix using steady state transcriptional expression data from the complete set of single-gene KOs. As mentioned above, the accessibility matrix includes both direct and indirect gene regulations, and thus provides an upper bound for the GRNs in the ensemble. Here, we follow the same procedure as that in TRaCE [12]. Briefly, for each technical replicate, we obtain the sample mean $\mu_{j}^{\prime}$ and standard deviation $s_{j}^{\prime}$ of the expression of gene $j$. Subsequently, we calculate the corrected sample mean $\mu_{j}$ and standard deviation $s_{j}$ by excluding the expression data of gene $j, g_{i j}$ that differ from $\mu_{j}^{\prime}$ by more than $z_{\text {cutoff }} s_{j}^{\prime}$. Using $\mu_{j}$ and $s_{j}$, we then compute the z-scores $z(i, j)=\frac{g_{i, j}-\mu_{j}}{s_{j}}$ which indicates the differential expression of gene $j$ in the $\mathrm{KO}$ of gene $i$. Finally, we average the $\mathrm{z}$-scores over the technical replicates to give the overall z-score matrix $Z(i, j)$, based on which we obtain the accessibility matrix by the following criteria:

$$
\operatorname{Acc}(i, j)=\left\{\begin{array}{ccc}
1 & \text { if } & |Z(i, j)|>Z_{\text {threshold }} \\
0 & \text { if } & |Z(i, j)| \leq Z_{\text {threshold }}
\end{array}\right.
$$

In this study, we use the accessibility matrix from the single-gene KO data as the initial ensemble upper bound $G^{U}$, which will later be refined by incorporating steady state gene expression data from optimally designed $\mathrm{KO}$ experiments. In the case studies, we employed $z_{\text {cutoff }}=3$ and $z_{\text {threshold }}=2$ following the recommendations in $\mathrm{TRaCE}[12]$. In contrast to TRaCE, we also set a sign for each edge (non-zero element in $A c c$ ) in the upper bound $G^{U}$ as follows:

$$
S(i, j)=\left\{\begin{array}{lll}
+ & \text { if } & Z(i, j) \leq 0 \\
- & \text { if } & Z(i, j)>0
\end{array}\right.
$$

\section{Construction of initial lower bound}

In TRaCE, the lower bound of the ensemble $G^{L}$ came from applying ConTREx (condensation, transitive reduction, and expansion) to the upper bound $G^{U}$ above without considering the signs [12]. Briefly, the upper bound $G^{U}$ was first condensed by lumping nodes in directed cycles, to give a DAG of strongly connected components. The subsequent transitive reduction involved removing from this DAG, any edge $(i, j)$ for which there exist a directed path from node $i$ to node $j$ not involving this edge. Finally, in order to get the lower bound $G^{L}$, we expanded the strongly connected components of directed cycles, during which any edges incident to cycles were removed, except those between nodes in a two-node cycle. Consequently, except when $G^{U}$ is a DAG, $G^{L}$ may no longer share the same accessibility matrix as $G^{U}$. However, the set of uncertain edges defined by the resulting $G^{U}$ and $G^{L}$ appropriately include edges in cycles with more than two nodes, as there exist ambiguity in either the existence or the direction of these edges.

Extending the transitive reduction procedure to a signed digraph means that we should remove any edge $(i, j)$ for which there exist a directed path from node $i$ to node $j$ not involving the edge $(i, j)$ and the cumulative product of the edge signs on this path is equal to the sign of the edge $(i, j)$. This simple procedure may not work when $G^{U}$ contains a negative cycle (a directed cycle with an odd number of negative edges), since the cumulative sign of such a cycle alternates depending on how many times one traverses through it. A recent study comparing different ways to obtain the transitive reduction of a signed GRN digraph (with and without cycles) recommended a simple procedure called Local Transitive Reduction (LTR) [14]. In the following, we have adapted LTR to generate the lower bound signed digraph $G^{L}$ for TRaCE+.

In LTR, an edge $(i, j)$ representing the regulation of gene $j$ by gene $i$, is removed when the effect of gene $i$ on gene $j$ can be explained by an indirect regulation involving at least one other gene. Such indirect regulation should explain not only the mode of the gene regulation (i.e. the sign of $(i, j))$ but also the strength of the regulation. Here, the strength of gene regulations is quantified by the weighting factor $W(i, j)$ (for each edge $(i, j)$ in $\left.G^{U}\right)$, which equals to the magnitude of the correlation coefficient between gene $j$ and gene $i$, averaged 
over the technical replicates. In the calculation of $W(i, j)$, we exclude data from gene $j \mathrm{KO}$ experiment since the differential expression of gene $j$ in this experiment may not reflect the effect of gene $i$ on gene $j$.

Adapting the LTR procedure, we first equate the two bounds $\left(G^{L}=G^{U}\right)$, and subsequently remove any edge $(i, j)$ in $G^{L}$ for which there exists a path of length 2 in $G^{U}$ explaining the edge $(i, j)$, or more precisely there exists a node $k$ with $(i, j),(i, k),(k, j) \in G^{U}$ and $k \notin\{i, j\}$, such that

a. the directed path $i, k, j$ is sign consistent with $(i, j)$, i.e. $S(i, j)=S(i, k) S(k, j)$.

b. the weight of the edge $(i, j)$ satisfies $w_{\text {cut }} W(i, j)<$ $W(i, k) W(k, j)$, where $w_{\text {cut }} \in[0,1]$ is a cutoff ratio.

The first condition requires the overall sign of the indirect regulation to equal to the sign of the gene regulation $(i, j)$. Meanwhile, the second condition requires the overall strength of the indirect regulation, which is assumed to accrue multiplicatively, to exceed a prescribed cutoff fraction of the strength of the edge $(i, j)$. Note that by setting $w_{\text {cut }}=0$, we effectively ignore the contribution of the edge weights. By considering only directed paths of length 2 , the procedure above avoids the problem associated with negative cycles since any path going through a cycle more than once would necessarily have a length longer than 2 . In addition, LTR does not require condensation and expansion steps as in TRaCE's ConTREx. Unlike the original version of LTR, here we do not check whether a removal of an edge would change the outcome of previous edge removals, and as a result, $G^{L}$ may not have the same accessibility relationships as $G^{U}$. While implementing the check would lead to fewer uncertain edges, it would also cause more false positive errors that could neither be corrected by additional data nor new experiments (see Discussion).

Figure 2 illustrates the computations of the lower bound $G^{L}$ in TRaCE and in TRaCE+ (using $w_{c u t}$ of 0.3 ) for the wild-type GRN $G_{\varnothing}^{+}$in Fig. 1a. The comparison showed that accounting for signs and weights of the edges could lead to a higher retention of true edges in $G^{L}$, and thus to fewer uncertain edges. This difference demonstrated that some information could be lost by disregarding edge signs in the GRN inference, as done in TRaCE.

\section{Ensemble bounds update}

The ensemble bounds update algorithm allows the incorporation of transcriptional expression data from gene $\mathrm{KO}$ experiments beyond single-gene KOs. The update follows an iterative procedure involving (1) evaluation of separatoids for uncertain edges, (2) determination of verifiable uncertain edges, and (3) refinement of the ensemble bounds. We previously introduced the concept of separatoid based on a simple premise, as illustrated below. Consider the ensemble upper and lower bounds in Fig. 2c and d, which define three uncertain edges (A, $D),(A, E)$ and $(C, E)$. Here, the verification of the uncertain edge (A, D) will be simple if we delete gene $C$ from the network. More specifically, in the background of gene $\mathrm{C} \mathrm{KO}$, we can verify the existence and sign of the edge $(A, D)$ based on whether a perturbation to gene $A$ causes a differential expression of gene $\mathrm{D}$ in the appropriate direction. In this case, gene $C$ is a separatoid of the uncertain edge $(\mathrm{A}, \mathrm{D})$.

More generally, we define a separatoid of an uncertain edge $(i, j) \in E_{U}$, denoted by $\operatorname{Sep}(i, j)$, as the set of nodes whose removal would eliminate any directed path of length 2 or longer from node $i$ to node $j$ [15]. The

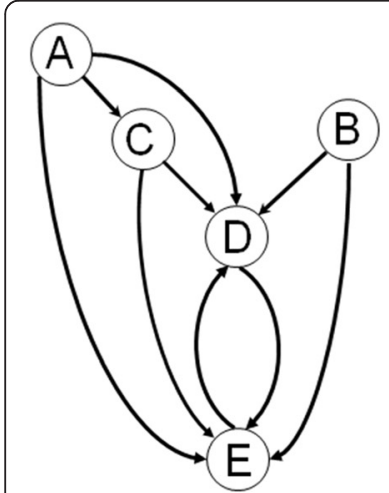

(a)
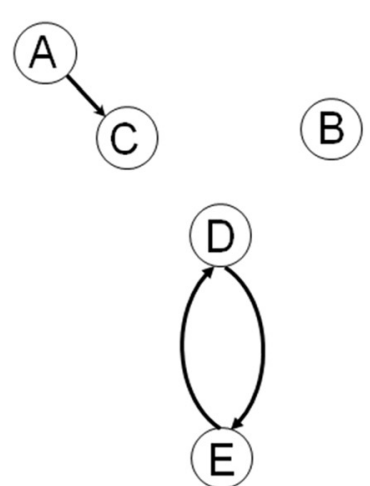

(b)

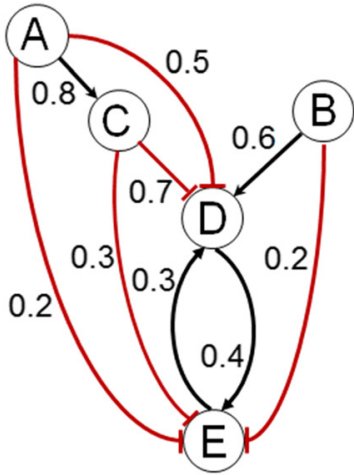

(c)

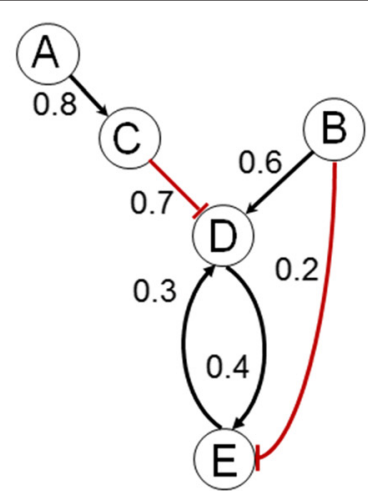

(d)

Fig. 2 Comparison of ensemble upper and lower bounds obtained from single-gene KO data by (a and b) TRaCE and (c and $\mathbf{d}$ ) TRaCE+ (with randomly assigned weights). The true GRN is shown in Fig. 1a. (a and $\mathbf{c}$ ) Ensemble upper bound $G^{U}$. (b and $\mathbf{d}$ ) Ensemble lower bound $G^{L}$. Note that the lower bound from TRaCE is not the transitive reduction of the upper bound due to the existence of a cycle between $D$ and $E$ 
deletion of genes in $\operatorname{Sep}(i, j)$ would therefore give the GRN $G_{\operatorname{Sep}(i, j)}$ where the only remaining directed path from gene $i$ to gene $j$ is the edge $(i, j)$ itself. Consequently, we can verify the existence of the uncertain edge $(i, j)$ by assessing whether gene $j$ is differentially expressed upon perturbing gene $i$ in the background of $\operatorname{Sep}(i, j)$ gene deletions. Returning to the ensemble bound in Fig. 2c and $\mathrm{d}$, we can find the separatoids of the uncertain edge (A, $E$ ), which are gene $C$, gene $D$ and the combination of genes $\mathrm{C}$ and $\mathrm{D}$; as well as the separatoid of the uncertain edge $(C, E)$, which is gene $D$.

As illustrated above, an uncertain edge may have more than one separatoid, while several uncertain edges may share a separatoid. Following our previous work [15], in the first step, we compute three separatoids for each uncertain edge $(i, j) \in E_{U}$ :

(1) $\operatorname{Sep}_{1}(i, j)=$ children of $i$ in $G^{U} \cap$ ancestors of $j$ in $G^{u, 0}$

(2) $\operatorname{Sep}_{2}(i, j)=$ descendants of $i$ in $G^{U, 0} \cap$ parents of $j$ in $G^{U}$

(3) $\operatorname{Sep}_{3}(i, j)=$ descendants of $i$ in $G^{U, 0} \cap$ ancestors of $j$ in $G^{u, 0}$

where $G^{U}$ is the most updated upper bound and $G^{U, 0}$ is the initial upper bound from single-gene KO data. The separatoids above are not the only separatoids for the uncertain edge $(i, j)$. We limit our analysis only to these separatoids because they are easy to compute. Finding all separatoids for a given edge constitutes finding the longest path between two nodes, which is a NP-hard problem [16]. In the illustration above, the three separatoids for the uncertain edge (A, E) are given by $\operatorname{Sep}_{1}(A, E)=\{C\}, \operatorname{Sep}_{2}(A, E)=\{D\}$, and $\operatorname{Sep}_{3}(A, E)=\{C, D\}$.

In the second step we identify among the set of input gene $\mathrm{KOs} \mathbf{V}_{\mathbf{K}}$ (including single-gene $\mathrm{KOs}$ ), pairs of $\mathrm{KO}$ experiments whose data would allow the verification of the edge $(i, j)$. More precisely, for each $(i, j) \in E_{U}$, we search for the pair of $\mathrm{KO}$ experiments $\left(V_{K O_{k}}, V_{K O_{k}} \mathrm{U}\right.$ $i) \in \mathbf{V}_{\text {ко }} \times \mathbf{V}_{\text {Kо }}$ such that $\operatorname{Sep}_{l}(i, j) \subset V_{K O_{k}}$ for any $l=1,2$, 3 , and $i, j \notin V_{K O_{k}}$. Following the definition of a separatoid above, if gene $j$ is differentially expressed between any of such pairs of $\mathrm{KO}$ experiments, then we have evidence supporting for the existence of the uncertain edge $(i, j)$

In the third step, for each uncertain edge $(i, j)$, we perform a (two-tailed) two-sample $t$-test with $\alpha=0.01$ to determine whether the expression of gene $j$ is significantly different between the $\mathrm{KO}$ of $V_{K O_{k}} \cup i$ in comparison to the $\mathrm{KO}$ of $V_{K O_{k}}$. In the case that we only find one pair of such experiments for an uncertain edge $(i, j)$, we remove this edge from the upper bound $G^{U}$ upon a failure to reject the null hypothesis in the $t$-test above. Otherwise, we add the edge $(i, j)$ to the lower bound $G^{L}$. Further, if the average expression of gene $j$ in the $\mathrm{KO}$ of $V_{K O_{k}} \cup i$ is lower (higher) than that in the $\mathrm{KO}$ of $V_{K O_{k}}$, we assign a positive (negative) sign to this edge.

In some cases, we may find more than one pair of $\mathrm{KO}$ experiments for an uncertain edge $(i, j)$. For each of these KO pairs, we again employ a two-sample $t$-test with $\alpha=$ 0.01 . The result of each $t$-test counts as a vote for the existence of the edge $(i, j)$ in the case of rejection of the null hypothesis, or a vote against the existence of this edge in the case of failure to reject the null hypothesis. If the votes against the edge exceed those for the edge, then we remove this edge from the upper bound $G^{U}$. Otherwise, we add the edge $(i, j)$ to the lower bound $G^{L}$. In case of a tie, we do not change the bounds, i.e. the edge $(i, j)$ remains uncertain. We also determine the edge sign by voting. Specifically, we set a positive (negative) sign when the average expression of gene $j$ in the $\mathrm{KO}$ of $V_{K O_{k}} \cup i$ is more frequently lower (higher) than that in the $\mathrm{KO}$ of $V_{K O_{k}}$, among the $\mathrm{KO}$ pairs giving confirmatory votes for the edge. When a tie occurs, we keep the original sign of the edge $(i, j)$ from $G^{U}$.

Once the ensemble bounds are updated, we recalculate the separatoids to reflect the changes brought by the additions and removals of edges to and from the bounds. We repeat the steps described above until we cannot find any suitable pairs of $\mathrm{KO}$ experiments for the remaining uncertain edges.

\section{Iterative gene regulatory network inference}

Recently, we proposed an iterative GRN inference procedure which combines TRaCE and an optimal design of gene $\mathrm{KO}$ experiments, called REDUCE (REDuction of UnCertain Edges) [15]. We demonstrated that this iterative procedure could resolve the underdetermined issue of the GRN inference, producing a unique GRN. $\mathrm{TRaCE}+$ can substitute TRaCE in this iterative inference to enable the inference of a signed digraph model of GRN. As shown in Fig. 3, the iteration starts with the construction of signed digraphs of the ensemble bounds using single-gene $\mathrm{KO}$ data. Based on these bounds, we optimize the next set of gene $\mathrm{KO}$ experiments using REDUCE. Briefly, REDUCE employs the edge separatoids and a constrained optimization to obtain the optimal set of gene KOs that would enable the verification of the maximum number of uncertain edges. The next step in the iteration is to carry out the optimized gene KOs experiments and obtain new transcriptional expression data. Subsequently, we feed the data back to TRaCE+ to update the ensemble bounds following the procedure described in the previous section. We repeat these steps until the ensemble bounds converge or do not improve further, or until a given quota on the number of $\mathrm{KO}$ experiments has been reached. 


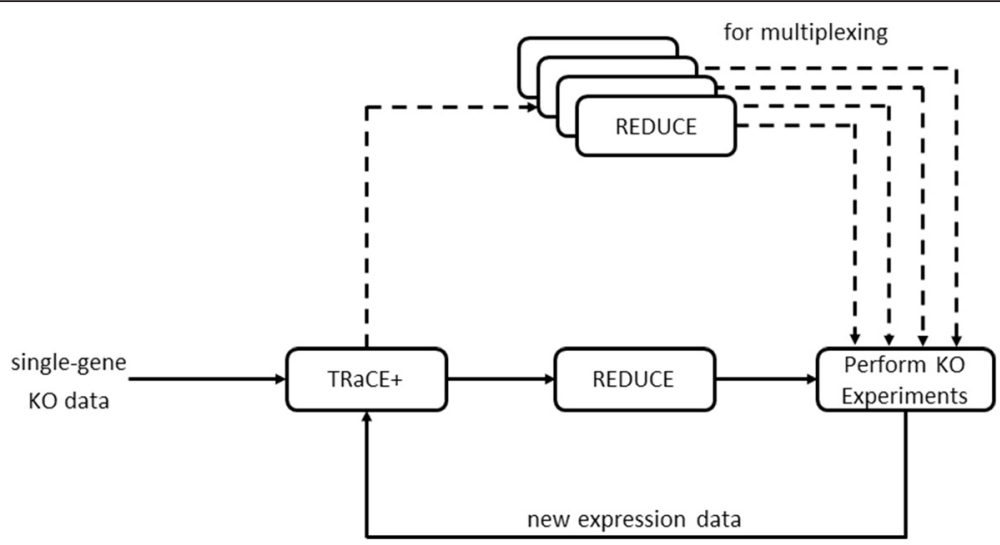

Fig. 3 Iterative GRN inference procedure. The procedure was adapted from [15]. Dashed arrows indicate the procedure for using multiplexing assay

If desired, one can also perform REDUCE multiple times in a given iteration to generate a large list of gene KO experiments (see Fig. 3, dashed arrows). This implementation is particularly suitable for using multiplexing assay technology. Here, at the end of one REDUCE optimization, we remove the set of verifiable uncertain edges from subsequent runs. We perform REDUCE until all uncertain edges become verifiable or until we hit constraint(s) on the optimization. When only a subset of these $\mathrm{KO}$ experiments could be performed, one should select gene KOs from the list above in the order that they are generated (since earlier runs of REDUCE are associated with more verifiable uncertain edges).

\section{Results}

\section{Case studies and performance evaluation}

In order to evaluate the performance of $\mathrm{TRaCE}+$, we applied the algorithms to the ensemble inference of Escherichia coli GRN from single-gene $\mathrm{KO}$ experiments, and to the iterative inference of 100-gene gold-standard GRNs from DREAM 4 in silico network inference challenge $[17,18]$. For each $\mathrm{KO}$ experiment, we generated 10 replicates of in silico (simulated) gene $\mathrm{KO}$ data using the benchmark GRN data generator GeneNetWeaver with the default parameters [17]. GeneNetWeaver uses a thermodynamic-based model of transcription and translation under independent and/or synergistic regulations [19]. The model consists of a system of stochastic differential equations (chemical Langevin equations), where the rates of change of concentrations for mRNA and protein are described by

$$
\begin{gathered}
F_{i}^{R N A}(x, y)=\frac{d x_{i}}{d t}=m_{i} f_{i}(y)-\lambda_{i}^{R N A} x_{i} \\
F_{i}^{\text {Prot }}(x, y)=\frac{d y_{i}}{d t}=r_{i} x_{i}-\lambda_{i}^{\text {Prot }} y_{i}
\end{gathered}
$$

where, $x_{i}$ and $y_{i}$ are the mRNA and protein concentrations for gene $i$, respectively, $t$ is time, $m_{i}$ is the maximum transcription rate, $r_{i}$ is the translation rate constant, $\lambda_{i}^{R N A}$ and $\lambda_{i}^{\mathrm{Pr} o t}$ are the RNA and protein degradation rate constants, respectively. The function $f_{i}(y)$ describes the regulations of the transcription of gene $i$ by different TF proteins. For example, the activation (positive regulation) of gene $k$ expression by the protein product of TF gene $j$ is described by a function $f_{k}(y)$, whose value increases with increasing value of $y_{j}$. Further, in GeneNetWeaver, both the production and degradation of RNA and proteins are subjected to intrinsic stochastic noise, modeled as a random Wiener process [17]. Additionally, log-normal measurement noise is added to the simulated expression data [20]. In the case studies below, the $\mathrm{KO}$ of a gene $i$ is simulated by setting the maximum transcription rate $m_{i}$ to zero.

The quality of the ensemble bounds was assessed by using true positive rate, total distance and Jaccard distance with respect to the reference GRNs. The true positive rate (TPR) was calculated as the ratio between the number of edges in the reference GRN that were correctly identified in the lower bound $G^{L}$ and the total number of edges in the reference network $G_{\varnothing}^{+}$, or more precisely:

$$
T P R=\frac{N\left(E\left(G^{L}\right) \cap E\left(G_{\varnothing}^{+}\right)\right)}{N\left(E\left(G_{\varnothing}^{+}\right)\right)}
$$

where $N\left(E\left(G_{\varnothing}^{+}\right)\right)$denotes the cardinality of the set $E\left(G_{\varnothing}^{+}\right)$. Meanwhile, the total distance between the ensemble bounds and the reference GRN was computed as follows:

$$
T D=\frac{N\left(E\left(G^{U}\right) \cup E\left(G^{L}\right) \cup E\left(G_{\varnothing}^{+}\right)\right)-N\left(E\left(G^{U}\right) \cap E\left(G^{L}\right) \cap E\left(G_{\varnothing}^{+}\right)\right)}{N\left(E\left(G_{\varnothing}^{+}\right)\right)}
$$

A higher TD value indicates larger uncertainty in the GRN inference (i.e. worse inferability). Finally, the Jaccard distance (JD) was evaluated using the following formula: 


$$
J D\left(G_{1}, G_{2}\right)=\frac{N\left(E\left(G_{1}\right) \cup E\left(G_{2}\right)\right)-N\left(E\left(G_{1}\right) \cap E\left(G_{2}\right)\right)}{N\left(E\left(G_{1}\right) \cup E\left(G_{2}\right)\right)}
$$

The JD gives a measure of similarity between two digraphs $G_{1}$ and $G_{2}$. A JD of 1 indicates that the two digraphs have no common edges and a JD of 0 implies that the two digraphs share the same set of edges. In the case studies, we evaluated the JDs between $G^{U}$ and $G_{\varnothing}^{+}$, as well as between $G^{L}$ and $G_{\varnothing}^{+}$. When dealing with ensemble bounds in the form of signed digraphs such as those generated by $\mathrm{TRaCE}+$, the intersections among the sets of edges in the evaluations of TPR, TD and JD were done by respecting the sign of the edges (i.e. edges of unequal signs were not counted).

\section{Ensemble inference of $E$. Coli GRN}

In this case study, we used the signed digraph of $E$. coli GRN from GeneNetWeaver, containing 1565 genes and 3758 regulatory interactions [17]. We generated in silico data for the complete set of single-gene KOs as described in Methods. Using this dataset, we constructed unsigned digraph ensemble bounds using TRaCE [12] and signed digraph bounds using TRaCE+. For TRaCE+, we also studied how the ensemble bounds, particularly $G^{L}$, depend on $w_{\text {cut }}$ by varying this parameter between 0 and 1 (at 0.1 increments). We compared the performance of TRaCE and TRaCE+ according to the TPRs, TDs and JDs of the resulting bounds as described in Methods. The comparison in Fig. 4 shows that TRaCE+ could provide ensemble bounds with higher TPRs and lower JDs and TDs (of the $G^{U}$ and $G^{L}$ from $G_{\varnothing}^{+}$) than TRaCE. These trends demonstrated TRaCE+'s ability to extract information contained in the gene regulatory signs that were disregarded by TRaCE. The TDs of the bounds from TRaCE+ generally improved with higher values of $w_{c u t}$, but the improvements reached a plateau after $w_{\text {cut }}$ of 0.4. However, the lower bounds from $\mathrm{TRaCE}+$ slightly worsened with increasing $w_{\text {cut }}$. The JDs between the upper bound and the reference network differed little between TRaCE+ and TRaCE due to the consideration of edge signs in computing JDs for the upper bounds from TRaCE+. As expected, the upper bound from $\mathrm{TRaCE}+$ did not vary with $w_{\text {cut }}$.

\section{Iterative GRN inference of DREAM4 100-gene networks}

In this case study, we applied the iterative GRN inference using either $\mathrm{TRaCE}$ or $\mathrm{TRaCE}+$ to the five signed digraph gold-standard networks from DREAM 4 100gene in silico network inference challenge [17, 18]. At the start of the iterative procedure, we simulated the complete set of single-gene $\mathrm{KO}$ data as described in Methods. Figure $5 \mathrm{a}$ and $\mathrm{b}$ show respectively the TPRs and TDs of the ensemble bounds. According to the TDs and TPRs, the ensemble bounds from TRaCE+ consistently outperformed those from TRaCE regardless of the parameter $w_{\text {cut }}$. Here, TDs and TPRs improved slightly with increasing $w_{\text {cut }}$. Figure $5 \mathrm{c}$ and d provide the JDs of the ensemble bounds from TRaCE and from TRaCE+ with different $w_{c u t}$ values. Like in the E. coli case study, the JDs of the ensemble upper bounds did not differ significantly between TRaCE and TRaCE+, nor did they depend on $w_{\text {cut }}$. The JDs of the lower bounds from $\mathrm{TRaCE}+$ were mostly better than those from TRaCE, where the best JDs corresponded to $w_{\text {cut }}$ values between 0.2 and 0.4. In the following, we compared the performance of the iterative inference using $\mathrm{TRaCE}$ and using

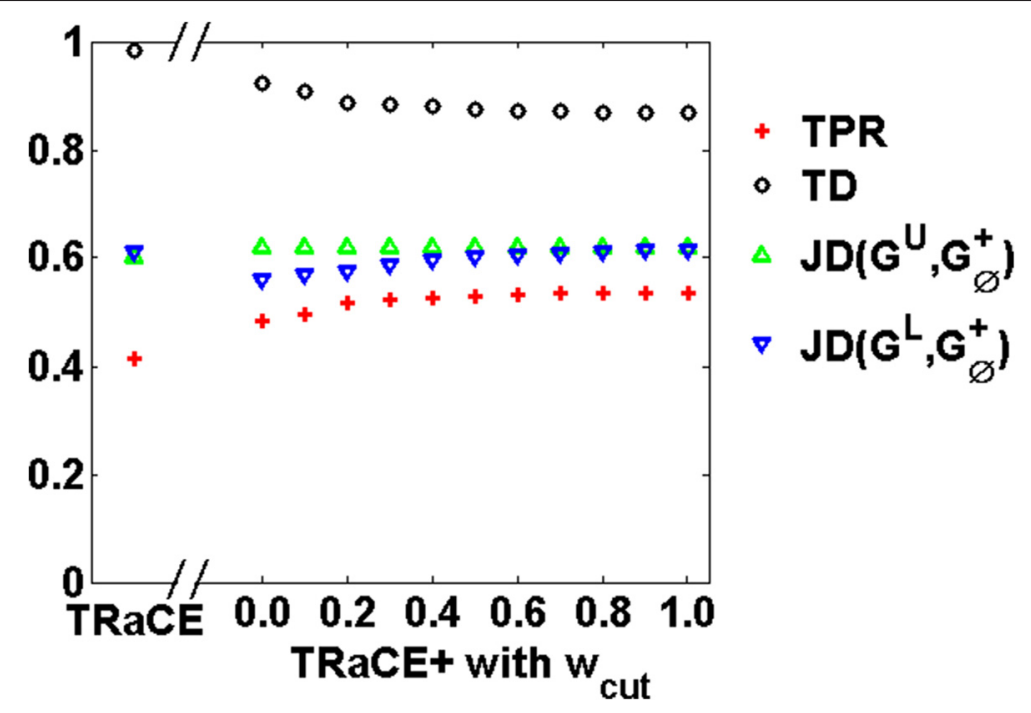

Fig. 4 Ensemble bounds from TRaCE and TRaCE+ for E. coli GRN: True positive rate (TPR), total distance (TD) and Jaccard distance (JD) 


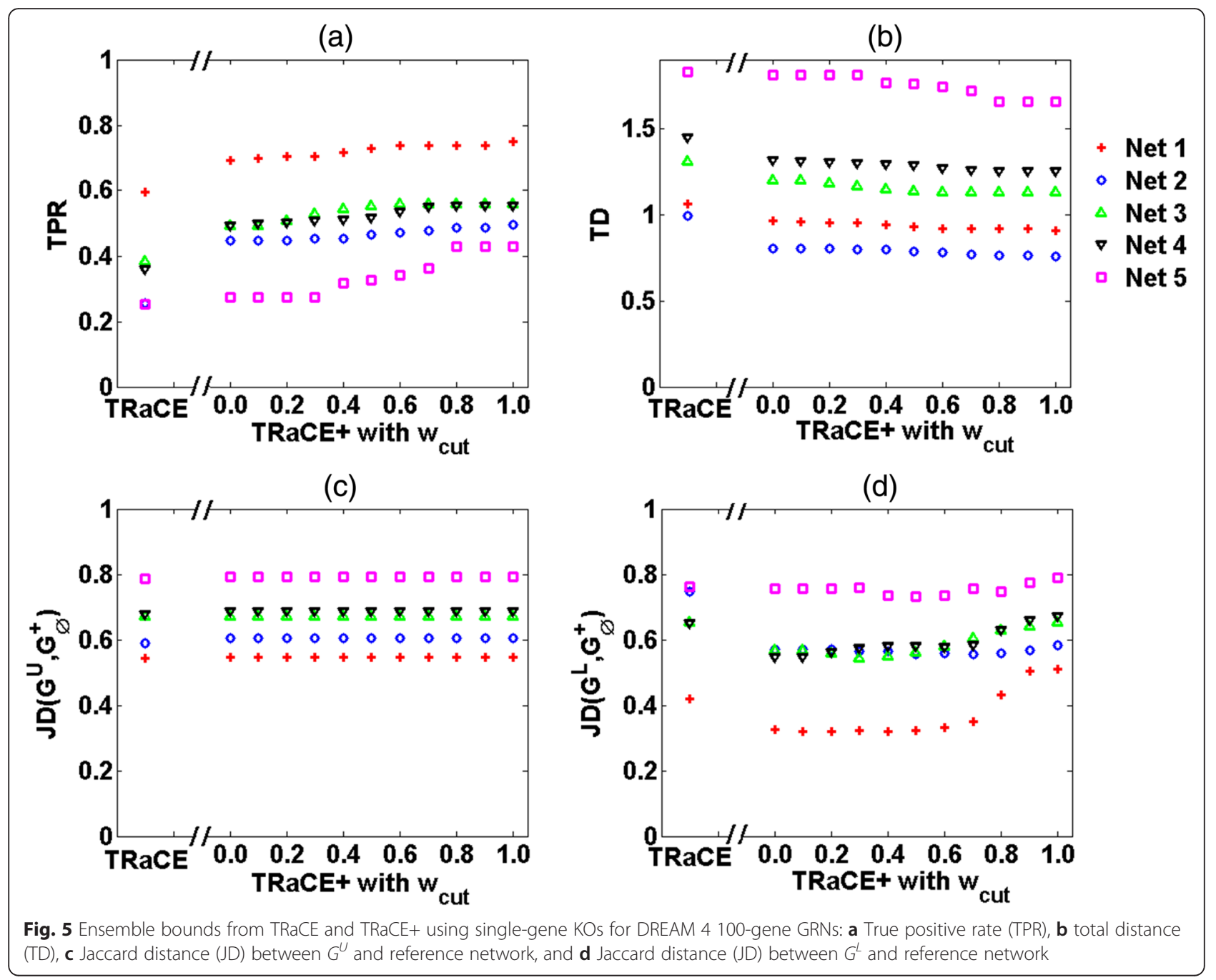

$\mathrm{TRaCE}+$ with a $w_{\text {cut }}$ of 0 (i.e. ignoring edge weights) and an intermediate $w_{\text {cut }}$ of 0.3 .

In the implementation of REDUCE, we put a constraint on the maximum number of genes in the optimal $\mathrm{KO}$ experiments. We started with a maximum of 2 genes, and incremented this constraint by 1 when the optimization within REDUCE could not produce any feasible solution. We again employed GeneNetWeaver to generate in silico data for the optimal $\mathrm{KO}$ experiments. We performed the iterative procedure until the ensemble bounds converged.

For all of the five gold-standard GRNs, the iterations terminated in the convergence of the ensemble bounds, i.e. we obtained a unique GRN. Figure 6 shows the TPRs, TDs and JDs of the inferred GRNs, as well as the total number of $\mathrm{KO}$ experiments required (excluding single-gene KOs). The iterations using TRaCE $+\left(w_{\text {cut }}=0\right.$ and $w_{c u t}=0.3$ ) produced slightly better GRNs than TRaCE in terms of TPRs and JDs. Of course, the edges in the GRNs from TRaCE+ had signs, while those from TRaCE did not. More importantly, the iterations using
$\mathrm{TRaCE}+$ required much fewer $\mathrm{KO}$ experiments to reach convergence than TRaCE $\left(p=0.013 w_{\text {cut }}=0\right.$ and $p=0.027$ for $w_{\text {cut }}=0.3$ ), by as much as $19 \%$. This trend signified the ability of $\mathrm{TRaCE}+$ to extract more information from the data. Table 1 further compares the number of iterations and the highest number of genes involved the $\mathrm{KO}$ experiments. The numbers of iterations using TRaCE+ were generally lower than using $\mathrm{TRaCE}$.

As a further comparison, we also generated in silico data for the complete set of double-gene KOs, a total of 4,950 KO experiments. We used this dataset to update the ensemble bounds initially constructed using single-gene KOs. As shown in Table 2, only a small fraction of the double-gene $\mathrm{KO}$ experiments were useful for verifying uncertain edges, and a number of uncertain edges still remained after the ensemble bound update. Figure 7 gives the TPRs, TDs and JDs of the ensemble bounds. Compared to TRaCE, the bounds update considering edge signs in TRaCE+ led to better TPRs $\left(p=0.02\right.$ for $w_{\text {cut }}=0$, and $p=0.016$ for $w_{\text {cut }}=0.3$ ). However, the differences in 


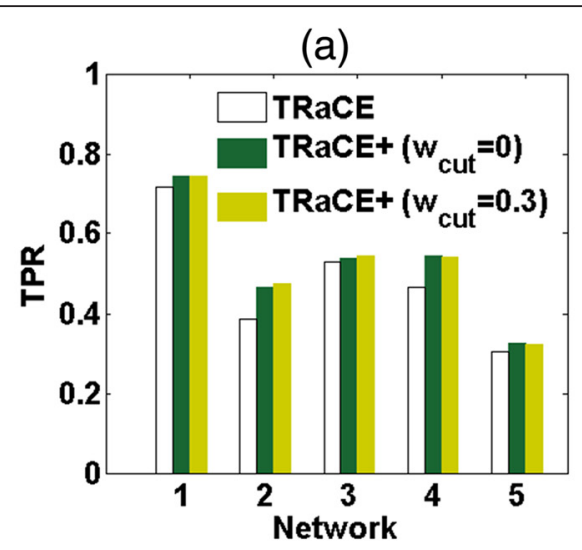

(c)

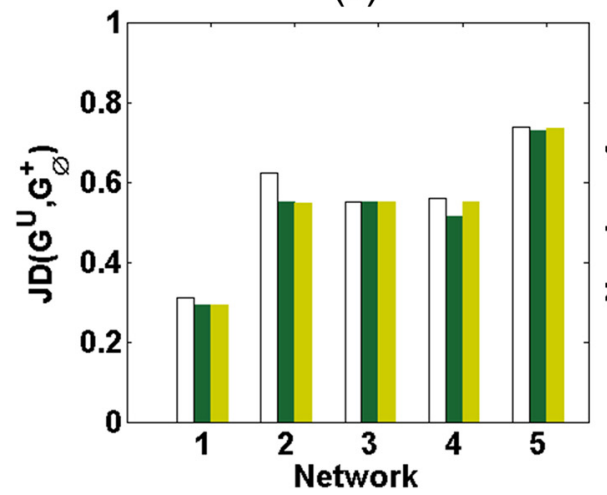

(b)

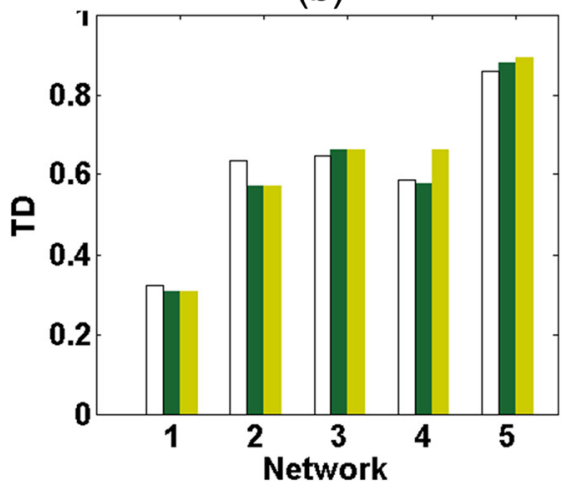

(d)

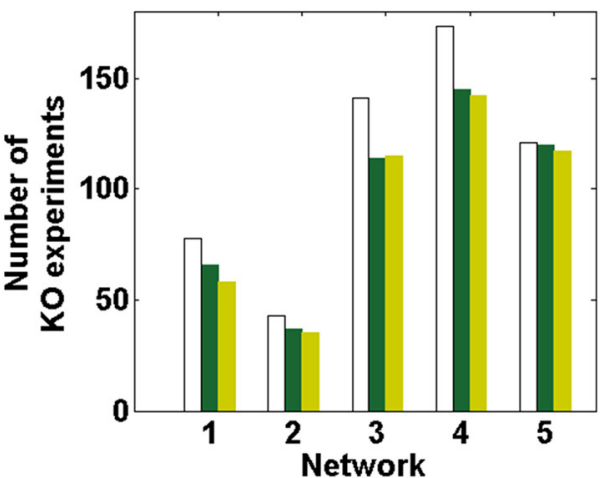

Fig. 6 Comparison of iterative GRN inference using TRaCE and TRaCE+ ( $w_{\text {cut }}=0$ and $\left.w_{\text {cut }}=0.3\right)$. a True positive rate (TPR), b total distance (TD), $\mathbf{c}$ Jaccard distance (JD), d total number of $\mathrm{KO}$ experiments

the TDs and JDs between TRaCE and TRaCE+ were not significant.

Finally, we performed the iterative procedure using $\mathrm{TRaCE}$ and using TRaCE + with $w_{\text {cut }}=0$ and $w_{\text {cut }}=0.3$, where we implemented multiplexed REDUCE to generate a large number of $\mathrm{KO}$ experiments. We again fixed the maximum number of genes in the $\mathrm{KO}$ experiments at each iteration, beginning with 2 and incrementing this limit by 1 when multiplexed REDUCE could not generate any feasible $\mathrm{KO}$ experiments. For all gold-standard networks, the iterations generated a unique GRN (i.e. the

Table 1 Iterative inference of DREAM4 100-gene gold-standard GRN using TRaCE and TRaCE $+\left(w_{\text {cut }}=0\right.$ and $\left.w_{\text {cut }}=0.3\right)$ : Number of iterations and maximum number of genes in $\mathrm{KO}$ experiments

\begin{tabular}{|c|c|c|c|c|c|c|}
\hline \multirow{2}{*}{$\begin{array}{l}\text { Gold- } \\
\text { standard } \\
\text { Network }\end{array}$} & \multicolumn{2}{|l|}{ TRaCE } & \multicolumn{2}{|c|}{ TRaCE $+\left(W_{\text {cut }}=0\right)$} & \multicolumn{2}{|c|}{ TRaCE $+\left(w_{\text {cut }}=0.3\right)$} \\
\hline & Iterations & Max KO & Iterations & Max KO & Iterations & Max KO \\
\hline 1 & 34 & 3 & 31 & 3 & 27 & 4 \\
\hline 2 & 21 & 2 & 18 & 2 & 17 & 2 \\
\hline 3 & 55 & 3 & 49 & 3 & 51 & 3 \\
\hline 4 & 80 & 3 & 70 & 3 & 68 & 4 \\
\hline 5 & 54 & 3 & 56 & 3 & 54 & 3 \\
\hline
\end{tabular}

ensemble bounds converged). Fig. 8 summarizes the quality of the ensemble bounds according to TPRs, TDs and JDs. The results closely resembled those from the iterations without multiplexing. Again, employing $\mathrm{TRaCE}+$ led to fewer total $\mathrm{KO}$ experiments than using $\operatorname{TRaCE}\left(p=0.011 w_{c u t}=0\right.$ and $p=0.006$ for $\left.w_{\text {cut }}=0.3\right)$. In comparison to the results without multiplexing in Table 1, Table 3 shows that multiplexing could reduce the number of iterations tremendously.

\section{Discussion}

In this work, we developed a new ensemble inference algorithm called $\mathrm{TRaCE}+$ for the identification of GRN structures in the form of a signed digraph. Unlike the traditional GRN inference, TRaCE+ produces upper and lower bounds of an ensemble of signed digraphs, describing the family of GRNs that are consistent with the gene accessibility relationships established by the input transcriptional expression profiles. Specifically, these bounds define the set of uncertain gene regulatory edges that could not be verified by the available data. The outputs of TRaCE+ are directly compatible with our recent optimal design of gene $\mathrm{KO}$ experiments called REDUCE and the accompanying iterative GRN procedure [15]. As 
Table 2 Ensemble bound update using double-gene KOs in TRaCE and TRaCE $+\left(w_{\text {cut }}=0\right.$ and $w_{\text {cut }}=0.3$ ): Number of informative double-gene KOs for verifying uncertain edges and number of remaining uncertain edges after ensemble bound update

\begin{tabular}{|c|c|c|c|c|c|c|}
\hline \multirow[t]{2}{*}{ Network } & \multicolumn{2}{|l|}{ TRaCE } & \multicolumn{2}{|l|}{ TRaCE $+\left(w_{\text {cut }}=0\right)$} & \multicolumn{2}{|l|}{ TRaCE $+\left(w_{\text {cut }}=0.3\right)$} \\
\hline & Informative experiments & Uncertain edges & Informative experiments & Uncertain edges & Informative experiments & Uncertain edges \\
\hline 1 & 36 & 15 & 24 & 20 & 20 & 21 \\
\hline 2 & 27 & 1 & 24 & 1 & 22 & 1 \\
\hline 3 & 61 & 37 & 52 & 30 & 50 & 27 \\
\hline 4 & 49 & 81 & 43 & 67 & 27 & 92 \\
\hline 5 & 46 & 37 & 44 & 37 & 44 & 37 \\
\hline
\end{tabular}

shown in the case study using 100-gene gold-standard GRNs from DREAM 4 in silico network inference challenge, by iterating TRaCE+, REDUCE and performing optimized gene $\mathrm{KO}$ experiments, one can overcome the underdetermined issue of GRN inference and obtain a unique GRN in a relatively small number of iterations (especially when using multiplexing assay). Like TRaCE, a drawback of $\mathrm{TRaCE}+$ is that the procedure requires at the minimum the complete set of single-gene $\mathrm{KO}$ data, which could become prohibitive for large-scale GRNs. If the TFs are known, then the requirement reduces to single-gene KOs of the TF genes. Nevertheless, we expect that accelerating progress in high-throughput gene editing technology (e.g., CRISPR-Cas9) and RNA sequencing will soon make this requirement non-limiting.

The consideration of regulatory signs in TRaCE+ represents a significant advance over TRaCE, as the mode of the gene regulations (activation/repression) is very often an important aspect in the applications of GRN. For example, when the inferred GRN is used in finding treatment of diseases or in drug discovery, the precise knowledge on the modes of the gene regulations matters tremendously. At the same time, the computational challenge arising from accounting the signs of the regulatory

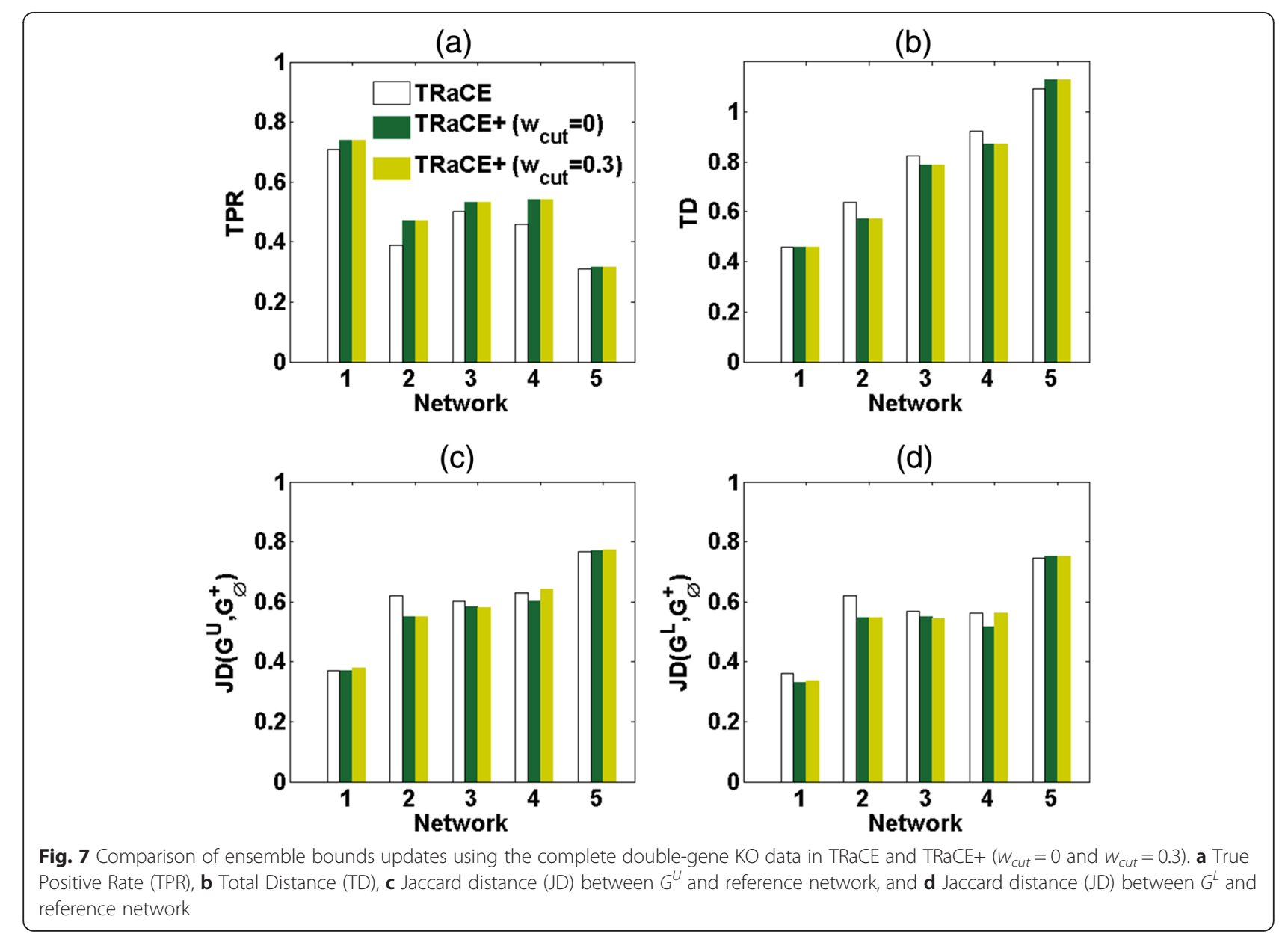


(a)

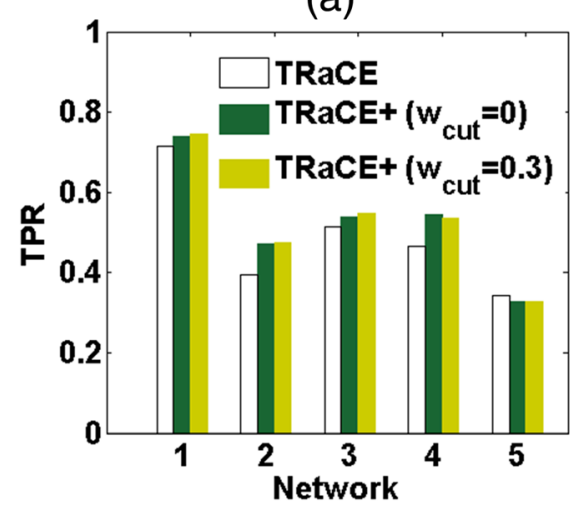

(c)

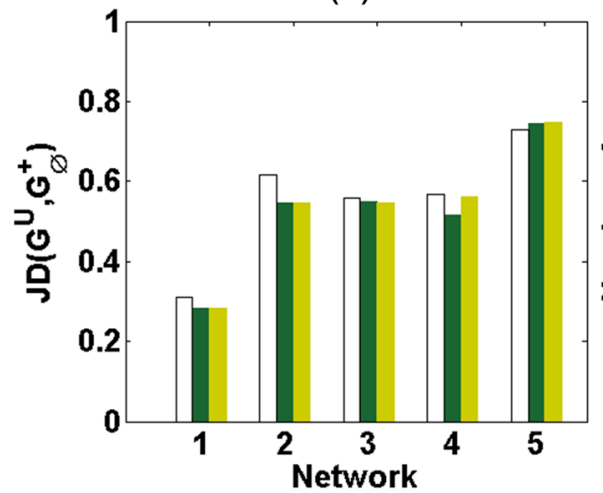

(b)

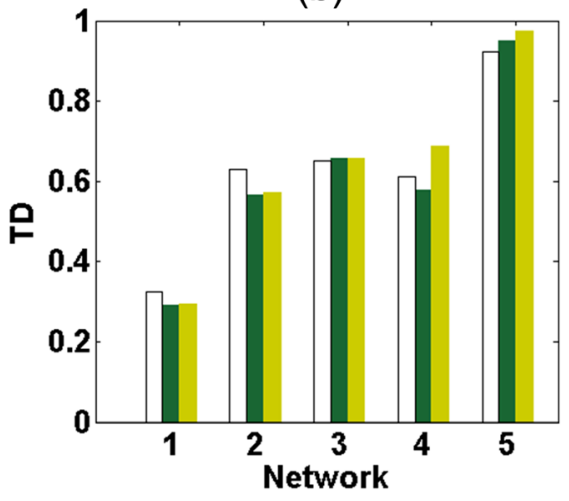

(d)

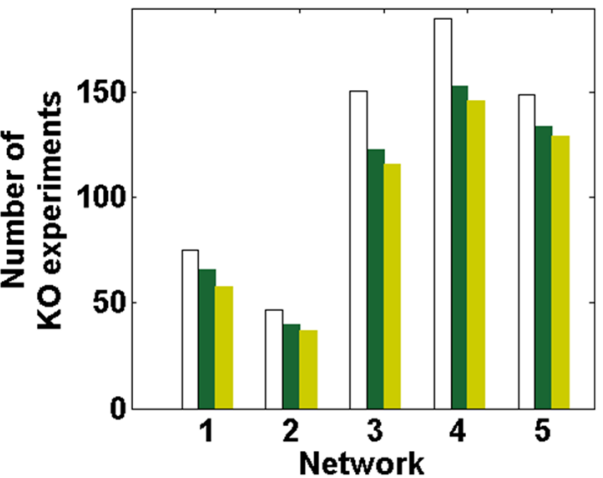

Fig. 8 Comparison of iterative GRN inference with multiplexing assay using TRaCE and TRaCE $+\left(w_{\text {cut }}=0\right.$ and $\left.w_{\text {cut }}=0.3\right)$. a True positive rate (TPR), b total distance (TD), c Jaccard distance (JD), and $\mathbf{d}$ total number of $\mathrm{KO}$ experiments

edges in the GRN digraph was not trivial. The issue of sign consistency could severely complicate performing transitive reduction [13], a key step in TRaCE+. Here, we adapted LTR to get around the issue in obtaining transitive reductions for GRNs with negative cycles. As demonstrated in the case studies, by taking the edge signs into account, $\mathrm{TRaCE}+$ can extract more information from the data than TRaCE. As a result, the numbers of uncertain edges in the ensemble from $\mathrm{TRaCE}+$ were consistently lower than those from TRaCE using the same set of $\mathrm{KO}$

Table 3 Iterative inference of DREAM4 100-gene gold-standard GRNs with multiplexing assay using TRaCE and using TRaCE+ $\left(w_{\text {cut }}=0\right.$ and $\left.w_{\text {cut }}=0.3\right)$ : Number of iterations and maximum number of genes in $\mathrm{KO}$ experiments

\begin{tabular}{|c|c|c|c|c|c|c|}
\hline \multirow[t]{2}{*}{ Net } & \multicolumn{2}{|l|}{ TRaCE } & \multicolumn{2}{|l|}{ TRaCE+ } & \multicolumn{2}{|c|}{$\mathrm{TRaCE}+(\mathrm{w})$} \\
\hline & Iterations & Max KO & Iterations & Max KO & Iterations & Max KO \\
\hline 1 & 7 & 3 & 5 & 3 & 6 & 4 \\
\hline 2 & 5 & 2 & 4 & 2 & 4 & 2 \\
\hline 3 & 10 & 3 & 11 & 3 & 12 & 3 \\
\hline 4 & 14 & 3 & 15 & 3 & 14 & 3 \\
\hline 5 & 11 & 3 & 12 & 4 & 13 & 4 \\
\hline
\end{tabular}

data (single-gene KOs). Furthermore, in the iterative inference, employing TRaCE+ led to significantly fewer total gene $\mathrm{KO}$ experiments to reach convergence than using TRaCE. While we used edge weights only for constructing the ensemble lower bound from the initial upper bound, these weights could also serve as a measure of confidence (likelihood) for the existence of an edge (as done in a previous method called TRANSWESD [13]).

There exist many reasons for errors to happen during the ensemble bounds construction and updates, including noise and bias in expression data as well as (type-I and type-II) errors in the statistical tests. Three types of errors can appear in the ensemble bounds from $\mathrm{TRaCE}+$. False negative (FN) errors involve regulatory edges in the reference GRN that do not appear in the upper bound $G^{U}$. Meanwhile, false positive (FP) errors pertain to regulatory edges in the lower bound $G^{L}$ that do not belong to the true network. Finally, incorrect sign (IS) errors refer to edges in the reference GRN that have the opposite signs in the upper bound. Among the three types of errors, our experience from the case studies showed that FNs were the most common errors while IS errors were the least common, typically affecting less than $1 \%$ of the edges in the reference 
networks. We further noted that the majority ( $>80 \%)$ of FN errors in the case studies were associated with fan-in motifs, where several genes regulated a common target gene. Here, knocking-out only one of the regulators might not cause any differential expression of the target gene due to compensation by the other regulator(s).

Once occurred, FP and FN errors could not be corrected during the iterative GRN inference since these errors affected edges that were not uncertain. In the second case study, the large majority of the errors in the inferred GRN were already present in the initial ensemble bounds constructed using single-gene KO data. Nevertheless, new FP and FN errors could also appear and accumulate over the iterations. By modifying the parameters in TRaCE+, including $z_{\text {cutoff }}, z_{\text {threshold }}, w_{\text {cut }}$ and $\alpha$, we can adjust the frequency of FPs and FNs. Lowering $z_{\text {cutoff }}$ and $z_{\text {threshold }}$ has the effect of reducing FN errors, but comes at the cost of higher FP errors and uncertain edges. We previously showed that $z_{\text {cutoff }}$ $=3$ and $z_{\text {threshold }}=2$ provide a good balance among FNs, FPs and uncertain edges [12]. Meanwhile, increasing $w_{\text {cut }}$ could reduce the number of uncertain edges, but also cause more FPs. On the other hand, lowering the parameter $\alpha$ in the ensemble bound update should reduce FPs at the trade-off of increasing FNs. In the ensemble bounds of E. coli and DREAM 4 GRNs from single-gene $\mathrm{KO}$ data, the frequency of FNs ranged between 24 and $56 \%$ (E. coli: $44 \%$ ), while the frequency of FPs varied between 2.8 and $13 \%$ (E. coli: $8.1 \%$ ) when using $w_{c u t}=0$. These frequencies were reported as a fraction of the number of edges in the reference GRN. Increasing $w_{\text {cut }}$ to 0.3 led to more FPs, especially for $E$. coli GRN (from 8.1 to $26 \%$ ). The increase in FPs by using $w_{\text {cut }}=0.3$ among DREAM 4 GRNs was however more modest $(2.9 \%-14.5 \%)$.

In this study, we focused specifically on transcriptional expression data from gene $\mathrm{KO}$ experiments when creating the ensemble of GRNs. Nevertheless, other types of information, such as transcription factor binding sites (TFBS) from chromatin immunoprecipitationsequencing (ChIP-seq), ChIP-chip, and/or cap analysis gene expression (CAGE) data, could also be used to refine the ensemble bounds. For example, we could verify uncertain edges emanating from a TF based on the existence or absence of its binding site in the promoter region of a target gene. In addition, TFBS could also be used to identify and correct FPs and FNs. However, the identification of the target genes of a TF based on ChIP-seq, ChIP-chip and CAGE data is not error-free. Thus, some care has to be taken to avoid accumulating different sources of errors. An integrative analysis of different types of data for ensemble GRN inference is out of the scope of this work, but is a topic of particular interest in our groups.

\section{Conclusion}

The inference of an ensemble of networks, rather than a single network, provides an avenue to cope with the underdetermined nature of the GRN inference from transcriptional expression data. In this work, we developed $\mathrm{TRaCE}+$ for the generation of upper and lower bound signed digraphs of GRN ensemble from gene KO data. TRaCE + significantly expanded the capability of our previous method TRaCE, enabling the inference of the mode of the gene regulations by considering the signs of the regulatory edges. As demonstrated in the case studies, TRaCE+ could extract more information from gene $\mathrm{KO}$ data than $\mathrm{TRaCE}$, and as a result, reduce the number of uncertain edges. When employed within an iterative inference procedure, $\mathrm{TRaCE}+$ required much fewer $\mathrm{KO}$ experiments to identify a unique GRN than $\mathrm{TRaCE}$, and slightly improved the quality of the reconstructed networks.

\begin{abstract}
Abbreviations
CAGE, cap analysis gene expression; ChIP, chromatin immunoprecipitation; ChIP-seq, chromatin immunoprecipitation - sequencing; ConTREx, condensation, transitive reduction, and expansion; DAG, directed acyclic graph; DNA, deoxyribonucleic acid; DREAM, dialogue on reverse-engineering assessment and methods; FN, false negative; FP, false positive; GRN, gene regulatory network; IS, incorrect sign; JD, jaccard distance; KO, knock-out; LTR, local transitive reduction; REDUCE, reduction of uncertain edges; RNA, ribonucleic acid; TD, total distance; TF, transcription factor; TFBS, transcription factor binding sites; TPR, true positive rate; TRaCE, transitive reduction and closure ensemble; TRANSWESD, transitive reduction for weighted signed digraphs
\end{abstract}

\section{Acknowledgements}

The authors would like to thank Andrea Pinna for explaining and helping with the implementation of local transitive reduction, and Lakshminarayanan Lakshmanan for his comments on the manuscript.

\section{Funding}

This project is supported by Swiss National Science Foundation (grants 137614 and 157154).

\section{Availability of data and material}

The MATLAB implementations of TRaCE+ and the in silico data used in this study are available at the following website: http://www.cabsel.ethz.ch/tools/ trace.html.

\section{Authors' contributions}

SMMU, SK and RG designed the study. SMMU simulated the datasets, wrote the codes, and analyzed the data. SH assisted in the implementation of LTR. SMMU, SK and RG wrote the manuscript. All authors read and approved the final manuscript.

\section{Competing interests}

The authors declare that they have no competing interests.

Ethics approval and consent to participate

No ethical approval or consent was required for this study.

\section{Author details}

${ }^{1}$ Institute for Chemical and Bioengineering, ETH Zurich, Zurich, Switzerland. ${ }^{2}$ Swiss Institute of Bioinformatics, Lausanne, Switzerland. ${ }^{3}$ Max Planck Institute for Dynamics of Complex Technical Systems, Magdeburg, Germany.

Received: 1 March 2016 Accepted: 12 June 2016

Published online: 24 June 2016 


\section{References}

1. Crick F. Central dogma of molecular biology. Nature. 1970;227:561-3.

2. Emmert-Streib F, Dehmer M, Haibe-Kains B. Gene regulatory networks and their applications: understanding biological and medical problems in terms of networks. Front cell deve biol. 2014;2:38.

3. Gardner TS, Faith JJ. Reverse-engineering transcriptional control networks. Phys Life Rev. 2005;2:65-88.

4. Markowetz F, Spang R. Inferring cellular networks-a review. BMC Bioinform. 2007;8 Suppl 6:S5

5. Bansal M, Belcastro V, Ambesi-Impiombato A, Di Bernardo D. How to infer gene networks from expression profiles. Mol Syst Biol. 2007;3:78.

6. Madhamshettiwar PB, Maetschke SR, Davis MJ, Reverter A, Ragan MA. Gene regulatory network inference: evaluation and application to ovarian cancer allows the prioritization of drug targets. Genome med. 2012;4:1-16.

7. Maetschke SR, Madhamshettiwar PB, Davis MJ, Ragan MA. Supervised, semisupervised and unsupervised inference of gene regulatory networks. Brief Bioinform. 2013;15:195-211.

8. Marbach D, Prill RJ, Schaffter T, Mattiussi C, Floreano D, Stolovitzky G. Revealing strengths and weaknesses of methods for gene network inference. Proc Natl Acad Sci U S A. 2010;107:6286-91.

9. Marbach D, Costello JC, Küffner R, Prill NMVRJ, Camacho DM, Allison KR, Consortium TD, Kellis M, Collins JJ, Stolovitzky G. Wisdom of crowds for robust gene network inference. Nat Methods. 2012;9:796-804.

10. Consortium TD. The DREAM Project. 2006.

11. Stolovitzky G, Prill RJ, Califano A. Lessons from the DREAM2 Challenges. Ann N Y Acad Sci. 2009:1158:159-95.

12. Ud-Dean SMM, Gunawan R. Ensemble Inference and Inferability of Gene Regulatory Networks. PLoS One. 2014;9:e103812.

13. Klamt S, Flassig RJ, Sundmacher K. TRANSWESD: inferring cellular networks with transitive reduction. Bioinformatics. 2010;26:2160-8.

14. Pinna A, Heise S, Flassig RJ, de la Fuente A, Klamt S. Reconstruction of large-scale regulatory networks based on perturbation graphs and transitive reduction: improved methods and their evaluation. BMC Syst Biol. 2013;7:73.

15. Ud-Dean SM, Gunawan R. Optimal design of gene knock-out experiments for gene regulatory network inference. Bioinformatics. 2015;32:875-883.

16. Bjorklund A, Husfeldt T, Khanna S. Approximating longest directed paths and cycles. Lect Notes Comput Sci. 2004;3142:222-33.

17. Schaffter T, Marbach D, Floreano D. GeneNetWeaver: in silico benchmark generation and performance profiling of network inference methods. Bioinformatics. 2011;27:2263-70.

18. Prill RJ, Saez-Rodriguez J, Alexopoulos LG, Sorger PK, Stolovitzky G Crowdsourcing network inference: the DREAM predictive signaling network challenge. Sci Signal. 2011;4:mr7.

19. Ackers $G K$, Johnson $A D$, Shea MA. Quantitative model for gene regulation by lambda phage repressor. Proc Natl Acad Sci. 1982;79:1129-33.

20. Stolovitzky G, Kundaje A, Held G, Duggar K, Haudenschild C, Zhou D, Vasicek T, Smith K, Aderem A, Roach J. Statistical analysis of MPSS measurements: application to the study of LPS-activated macrophage gene expression. Proc Natl Acad Sci U S A. 2005;102:1402-7.

\section{Submit your next manuscript to BioMed Central and we will help you at every step:}

- We accept pre-submission inquiries

- Our selector tool helps you to find the most relevant journal

- We provide round the clock customer support

- Convenient online submission

- Thorough peer review

- Inclusion in PubMed and all major indexing services

- Maximum visibility for your research

Submit your manuscript at www.biomedcentral.com/submit

) Biomed Central 